\title{
Shaping Attitudes Toward Science in an Introductory Astronomy Class
}

\author{
David Wittman \\ University of California, Davis, CA
}

September 5, 2021

At many universities, astronomy is a popular way for non-science majors to fulfill a general education requirement. Because general-education astronomy may be the only college-level science course taken by these students, it is the last chance to shape the science attitudes of these future journalists, teachers, politicians, and voters. Hobson ${ }^{1}$ recently reported on research indicating that general education requirements (often as little as a single class) are responsible for the higher measured level of science literacy in the US as compared to Europe.

Science and society will benefit from these students gaining a deeper understanding of the process of science, rather than focusing exclusively on its results. Once outside the university, these students will find themselves in a society in which two-thirds of adults (and $38 \%$ of college graduates!) believe that humans were created "pretty much in their present form at one time within the last 10,000 years." ${ }^{2}$ They should be equipped with the tools to refute or at least resist common anti-science attitudes such as "we can't know what happened in the distant past because no one was there to observe it." Astronomy, like evolutionary biology and geology, makes use of fossil evidence, so instructors have an opportunity to address this issue directly.

If shaping student attitudes is an important goal to us as instructors, then we should measure those attitudes before and after instruction, and consider the connection between attitude changes and classroom activities. I report here on pre- and post-instruction surveys of science attitudes in my general education astronomy course, and on classroom activities designed to influence those attitudes. The first step, well before instruction begins, is to design the attitude survey.

\section{Measuring attitudes}

Those who teach introductory physics should be aware that several attitude assessment instruments are readily available, including $\mathrm{CLASS}^{3}$ (Colorado Learning Attitudes about Science Survey), MPEX $^{4}$ (Maryland Physics Expectations Survey), and VASS ${ }^{5}$ (Views About Science Survey). Each of these probes attitudes toward learning, especially problem-solving, more heavily than attitudes 
toward science. VASS is somewhat stronger on the scientific process and the nature of scientific laws than the other two, and comes with a taxonomy ${ }^{6}$ which clearly lays out the motivation for each question. For example, in the epistemology section, the taxonomy states that "Science is a coherent body of knowledge about patterns in physical realities (real systems or phenomena), rather than a loose collection of particular empirical facts" and points to questions 5,6 , and 7 as specifically probing that issue. In a section labeled "readiness to learn" the taxonomy states that "science is learnable by anyone willing to make the effort, - not just by a few talented people" and points to question 29 as the relevant probe. Collectively, the statements in the VASS taxonomy provide an excellent summary of what is missing in the general publics attitudes toward science.

One should use an existing survey if at all appropriate, because they have been extensively field-tested over a range of institutions and instruction styles. However, none of the above are appropriate for a general education astronomy course, so I wrote a set of questions specifically for my course. I started with general science attitudes such as those mentioned above in the discussion of VASS. For example, students are asked to rate the statement "Science is largely about memorizing facts" on a Likert scale (strongly disagree to strongly agree on a scale of 1-5 or $a-e$ on a scantron form). I included several questions related to this attitude, because in a large lecture hall, in a nonmathematical course with no lab component (none of these parameters is under my control), it would be easy to miss the process of science and slip into merely describing the results. Another example, motivated in part by science coverage in the media which celebrates "discoveries" without considering the surrounding framework: "Scientists do experiments: (a) on what interests them the most; (e) on things that could verify or falsify theories." This is a forced-choice type of question, in which students must choose between the two options on a five-letter scale, with $a$ indicating complete agreement with the first option and $e$ indicating complete agreement with the second option.

Because this is an astronomy course, I added items relating to reconstructing the past and our place in the universe, for example "We can't know what happened in the distant past because we dont have any observations of that time" and "We live in a changing universe." A few questions are somewhat factual in nature, but relate strongly to attitudes about our place in the universe, e.g. "The Sun is a special star."

The list of nineteen questions on science attitudes appears below along with the results; ; a copy of the survey also appears at the end of this preprint and is available for anyone to use freely. I gave the survey at the start of the first day of class and at the end of the final day. Surveys are more conveniently delivered online, but instructors should weigh this convenience against the likelihood of getting online responses from a biased sample of more conscientious students (or offer extra credit and issue several email reminders). Because there is a good deal of dropping and adding courses in the first two weeks of the term, I asked students to write their student identification numbers on the survey so that I could rigorously match pre- and post-instruction. I assured them that their attitudes would not be studied individually, but writing their ID may nevertheless have put them on better behavior compared to a completely anonymous survey. 


\section{Shaping attitudes}

If writing the attitude survey helps clarify the goals, clear goals in turn guide the construction of classroom activities to further those goals. Activities and case studies are more likely to influence attitudes than simply lecturing about the nature of science, and making the nature of science a recurring theme is likely to have more impact than a single first-day activity. While I can't prove a causal relationship between these activities and attitude changes, I offer a few examples which instructors may find useful.

The first week, I give the students Ob-scertainers--little black boxes whose internal structures they must deduce from the sound of a ball bearing rolling around inside. They work in groups of two to form hypotheses, then groups double up to compare hypotheses and conduct additional tests. At the end, when I am no doubt expected to reveal the correct answer, I tell them that nature never reveals the correct answer to scientists. ${ }^{7}$ All we can do is obtain more data and refine our hypotheses, so I ask the students to suggest additional methods of doing so. They offered many creative and enthusiastic responses to this open-ended question.

The energy source of the Sun and the age of the solar system make a nice case study. In the late 1800s fusion was not yet known, and the largest energy source astronomers knew of was KelvinHelmholtz contraction, which could power the Sun for about 25 million years. Yet geologists were confident from the study of rocks that the Earth was of order 1 billion years old. What would you decide if this was all the information you had? Is the statement that "astronomers don't know of an energy source that can power the Sun for 1 billion years" enough to discredit the geological evidence? On the other hand, should we just assume from the geological evidence that there must be a source of power orders of magnitude beyond what is currently known? This relates not only to the tentative nature of scientific conclusions, but also the interconnectedness of all branches of science, and the ability to recognize and mentally process the existence of large gaps in our knowledge. The fact that astronomers later radically changed their views of the Suns power source is an indication of science's strength, not its inefficacy, a point that should serve students well when reading modern-day press releases which tend to overly emphasize challenges to established scientific views.

Finding hands-on activities that work well in a large lecture hall can be a challenge. Particularly well-suited is a demonstration of why photons take of order a million years to travel from the core of the Sun to its surface. A balloon is placed in the center of the audience and students are first told to pass it forward; it quickly leaves the audience. The balloon is reset in the center and this time students are told to pass it in random directions. It takes much longer to reach the edge of the audience. (This is one of those rare activities where the larger the lecture hall, the better.) I use the activity not only to demonstrate the idea, but to let the students participate in making and testing models and lead them to understand that scientists do the same thing on a more mathematical level.

Another good case study is the Shapley-Curtis debate over the scale of the universe: Are the spiral nebulae small objects within our own Galaxy, or are they large, distant "island universes" 
unto themselves? At the time of the formal debate in 1920, the evidence was conflicting, butand this is key to the nature of science-when solid new evidence was presented, the debate was over. Furthermore, an important piece of data against the "island universe" interpretation was later shown to have been incorrectly measured. This provides an excellent opportunity to discuss how scientists reach conclusions. I poll the class after presenting the original evidence, then after retracting some of the evidence, etc. This illustrates the conditional nature of scientific conclusions.

\section{Results}

Post-instruction, I first matched the pre and post tests to yield a set of $n=63$ students who had taken both. Next, I inverted the scores on questions for which the correct attitude was 1 or $a$ rather than 5 or $e$, so that a higher number is always considered better. In measuring the learning of content, it is standard to define the gain as posttest score minus pretest score, and normalized gain as (posttest-pretest)/(maximum-pretest), in other words, how much the students learned as a fraction of what they didn't already know. ${ }^{8}$ I adopt these definitions here, with the caveat that this survey deals with attitude changes which is not the same as learning content. For example, expert attitudes will not always be 5.0 because of some difficult forced choices, and should be determined by giving the same survey to professional scientists. This is a clear advantage of using an existing instrument such as VASS, which has been extensively field-tested in different populations, if it is appropriate to the course. However, the lack of an appropriate field-tested survey should not prevent instructors who care about attitudes from trying to measure them. The results cannot be interpreted as rigorously, but the process can lead to better teaching and learning.

I list the results below in descending order of normalized gain, expressed here as a percentage, in a recent term. In parentheses, I list the pre- and post-instruction average attitude.

$57 \%$ (4.44, 4.76) A bit of information is considered scientific when: (a) it is supported by evidence; (e) it is supported by eminent scientists.

$52 \%(3.30,4.19)$ Most of the atoms in your body were once inside a star.

$50 \%(4.14,4.57)$ If a scientist publishes an interesting new theory, other scientists usually: (a) compete to prove it wrong; (e) ignore it because it is not their theory.

$49 \%(3.47,4.22)$ We can't know what happened in the distant past because we don't have any observations of that time.

$41 \%(4.24,4.56)$ We live in a changing universe.

$39 \%(4.64,4.78)$ If a scientist publishes an interesting experimental result which is in error, the error is usually detected by: (a) no one; (e) other scientists attempting to reproduce the result.

$31 \%(3.56,4.02)$ Science is largely about memorizing equations. 
$29 \%(2.87,3.51)$ The Sun is a special star.

$27 \%(4.21,4.43)$ Science affects everyone's life.

$26 \%(3.29,3.74)$ We can't know what happened in the distant past because we can't do controlled experiments on it.

$24 \%$ (4.54, 4.65) An experimental result: (a) should be trusted because it is scientific; (e) should be questioned as to how it fits in with other experimental results.

$21 \%(3.43,3.76)$ Science is largely about memorizing facts.

$20 \%$ (4.06, 4.25) Scientists who created successful theories did it by: (a) having exceptional intelligence; (e) looking for patterns in data.

$14 \%(3.57,3.78)$ Science is largely about discovering patterns.

$10 \%(3.60,3.75)$ Anyone can understand the major themes of science if they try.

$9 \%(2.87,3.08)$ Astronomy is difficult because the physical laws that apply on Earth do not necessarily apply everywhere.

$0 \%(3.68,3.68)$ I want my tax money to help support science.

$-3 \%(3.70,3.65)$ Science is fun.

$-9 \%(3.43,3.29)$ Scientists do experiments: (a) on what interests them the most; (e) on things that could verify or falsify theories.

The typical per-question statistical uncertainty in gain (posttest-pretest) is 0.12 points. Thus the two declines are not statistically significant. Several attitudes improved by many times the statistical uncertainty, with a normalized gain of $~ 50 \%$, comparable to the typical normalized gain for subject matter in effective courses. ${ }^{9}$ Many other attitudes individually showed a gain of only a few times the statistical uncertainty, but collectively they represent a significant shift.

\section{Summary and conclusions}

It $i s$ possible to influence student attitudes for the better. Several previous studies have shown that attitudes tend to decline or remain unchanged after introductory physics and astronomy courses, ${ }^{4,9}$ with just a few small-scale counterexamples. ${ }^{10,11}$ However, these studies have focused more on attitudes toward learning. Attitudes toward science may be less deeply ingrained.

The results here are promising, but with no control group the efficacy of the instructional approach cannot be rigorously established. In retrospect, the process of preparing the attitude survey was key to the entire venture, as it forced me to reflect on and organize my objectives with respect to student attitudes. I encourage other instructors to engage in this process and see where it leads. 


\section{Notes}

${ }^{1}$ A Hobson, "The Surprising Effectiveness of College Scientific Literacy Courses", Physics Teacher 46, 404 (2008)

${ }^{2}$ The two-thirds figure includes those who rated the statement as "definitely true" or "probably true" in a USA Today/Gallup poll published June 7, 2007: http://www.usatoday.com/news/politics/2007-06-07-evolution-poll-results_N.htm?csp=? Polls do not ask specifically about the age of the Earth, but one can take this as a rough indicator of public attitudes. The 38\% figure for college graduates comes from a meta-analysis of several Gallup polls with slightly varying wording: http://www.gallup.com/poll/21811/ American-Beliefs-Evolution-vs-Bibles-Explanation-Human-Origins.aspx.

${ }^{3}$ W.K Adams et al., "A new instrument for measuring student beliefs about physics and learning physics: the Colorado Learning Attitudes about Science Survey," Phys. Rev. ST Phys. Educ. Res. 2, 010101 (2006); see also http://CLASS.colorado.edu

${ }^{4}$ Edward F. Redish et al., "Student Expectations in Introductory Physics," Am. J. Phys. 66, 212-224 (1998); see also http://www.physics.umd.edu/perg/expects/index.html

${ }^{5}$ I. Halloun \& D. Hestenes, "Views About Science Survey", Annual Meeting of the National Association for Research in Science Teaching. Saint Louis, Missouri (1996). ERIC Document No. ED394840. See also http://cresmet.asu.edu/prods

${ }^{6} \mathrm{http}: / /$ cresmet.asu.edu/prods/VASS-P204 Taxonomy.pdf

${ }^{7}$ Credit for this idea goes to Steve Shawl of Kansas University.

${ }^{8}$ R. R. Hake, "Interactive-engagement vs traditional methods: A six-thousand-student survey of mechanics test data for introductory physics courses," Am.J.Phys. 66, 64 (1998)

${ }^{9}$ M. Zeilik et al, "Conceptual astronomy. II. Replicating conceptual gains, probing attitude changes across three semesters", Am.J.Phys. 67, 923 (1999)

${ }^{10}$ A. Elby, Helping physics students learn how to learn, Am. J. Phys. 69, S54 (2001)

11 J. Marx and K. Cummings, "What Factors Really Influence Shifts in Students Attitudes and Expectations in an Introductory Physics Course?", AIP Conference Proceedings 883, 101 (2007) 
These questions are designed to measure the incoming attitudes of the class. Please answer honestly. You will not be identified individually. (We ask for your student ID on the scantron only so that we can look at attitudes of different groups.) Please do not mark the questions, so that the paper can be reused.

For questions which are simple statements, such as number 1, respond as follows:

1: strongly disagree

2: somewhat disagree

3: neither agree nor disagree

4: somewhat agree

5: strongly agree

For questions which have two alternatives, such as number 2, respond as follows:

1: strongly agree with alternative a rather than e

2: somewhat agree with alternative a rather than e

3: agree about the same with a or e

4: somewhat agree with alternative e rather than a

5: strongly agree with alternative e rather than a

1. Science is largely about memorizing facts.

2. Scientists do experiments:

(a) on what interests them the most

(e) on things that could verify or falsify theories

3. Scientists who created successful theories did it by

(a) having exceptional intelligence

(e) looking for patterns in data

4. Science is largely about memorizing equations.

5. A bit of information is considered scientific when:

(a) it is supported by evidence

(e) it is supported by eminent scientists

6. Anyone can understand the major themes of science if they try. 
7. Science is fun.

8. An experimental result:

(a) should be trusted because it is scientific.

(e) should be questioned as to how it fits in with other experimental results.

9. Science affects everyone's life.

10. Science is largely about discovering patterns.

11. If a scientist publishes an interesting experimental result which is in error, the error is usually detected by:

(a) no one

(e) other scientists attempting to reproduce the result

12. If a scientist publishes an interesting new theory, other scientists usually:

(a) compete to prove it wrong

(e) ignore it because it is not their theory

13. We live in a changing universe.

14. The Sun is a special star.

15. Astronomy is difficult because the physical laws that apply on Earth do not necessarily apply everywhere.

16. Most of the atoms in your body were once inside a star.

17. I want my tax money to help support science.

18. We can't know what happened in the distant past because we don't have any observations of that time.

19. We can't know what happened in the distant past because we can't do controlled experiments on it.

Thank you for completing this survey! 